Lepr. Rev. (1969) 40, 171-173

\title{
The Use of Plastazote to Accommodate Deformities in Hansen's Disease*
}

\author{
W. H. TUCK \\ Manager, Orthopaedic Appliance Workshop \\ Royal National Orthopaedic Hospital, Stanmore, Middlesex, England
}

\begin{abstract}
This paper gives some useful advice by an expert on the making of Plastazote insoles for leprosy patients with foot deformities and plantar ulceration, and describes the results achieved in 2 such severely disabled patients. Many readers who attended the Ninth Leprosy Congress in London will have seen the author demonstrating the speed and apparent ease with which Plastazote can be moulded.
\end{abstract}

It was early in 1967 that I discovered the use of Plastazote for orthopaedic purposes and my immediate reaction was to use this material for patients with leprosy. Many orthopaedic footwear manufacturers today are using Plastazote, and for grossly deformed feet this material has proved that it is not only light in weight and easy to mould and is something which has been sought for many years. Its introduction holds out great promise for the healing of trophic ulcers and in dealing with some gross deformities of the feet, particularly those with ulceration. Experience early showed that by using Plastazote in 1 in. $(2.5 \mathrm{~cm})$ thickness under ulcerated areas the ulcers soon became much smaller and in many cases healed completely.

My first encouragement outside my own hospital came from Dr. Paul Brand, who has dealt with leprosy patients in many parts of the world and who for years had been looking for a mouldable material which would be of help in dealing with these gross deformities of the feet. As soon as I had used Plastazote and found how useful it was, I told him about it, and received a reply saying that after he had used it he had seen for the first time a smile on his surgical bootmaker's face, which was very encouraging. Later, at the Ninth Leprosy Congress in London last year, Dr. Brand con-

*Received for publication 28 April, 1969. firmed that he was very pleased with the results achieved with Plastazote for his leprosy patients with foot deformities, and was using it in conjunction with sandals.

The following 2 cases of my own may be of interest. The first was that of a leprosy patient who had had considerable trouble with his footwear over a number of years. About half his left foot had been amputated and he had also lost all his toes and part of the metatarsals from the right foot. The soles of the feet were in very poor condition due to ulceration, and when his feet became anaesthetic he suffered considerable anxiety, to which was added worry about his future condition. He was also very concerned about the appearance of his footwear. From the age of 36 for a number of years he had worn surgical shoes with a full $\frac{1}{2}$ in. spongerubber insole, sponge-rubber metatarsal pads, and sponge-rubber toe-blocks and wedges built in to accommodate the deformity. These orders were repeated every year and sometimes his feet were in a fair condition and at other times they were worse. He had periods in hospital, where he underwent some further amputations, and in 1965 he had to be re-admitted because of discharge from the deformities. Progress was very slow and unsatisfactory, however, until in 1967 I started to use Plastazote supports for him. A great deal of care was taken to obtain good plaster casts of both his feet and to mould the Plastazote to the base of his feet, under 
pressure. The present position is that since wearing surgical boots with well made insoles and with Plastazote made to come up to the forefoot, the patient is now much happier about his condition. There is no sign of ulceration and the soles of the remainder of his feet look very healthy. In spite of his being a somewhat despondent type of patient, he seems to have completely accepted the fact that his feet are as well as they are ever likely to be and he is able to earn his living quite satisfactorily.

The second patient, also with leprosy, had had all his toes amputated with the exception of the great toe. For a number of years he suffered from ulceration underneath the head of the fifth metatarsal and the foot generally had rather an unhealthy look. During this time he had his shoes made for him, these being fitted in the usual way with a toe block. This is typical of the type of foot that invariably required (when only sponge rubber was available) a very good, thick, sponge-rubber insole and a varus wedge to throw the weight back on to the great toe, because usually these patients cannot obtain a good balance. The problem with this patient was that he wanted more acceptable footwear, so we made for him a pair of sandals of 1 in. $(2.5 \mathrm{~cm})$ Plastazote with elastic across the top and the heel piece also made of Plastazote. On the left side, to counteract slipping, we put some extra padding inside to throw the weight back. He is now able to wear this type of sandal in the house and also on holiday, and he considers this to be a very real advantage. For a patient with this type of

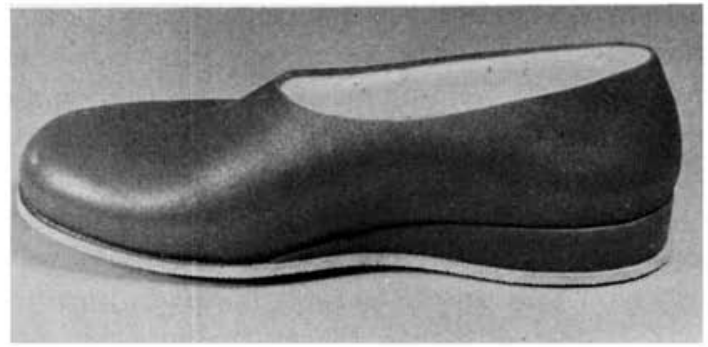

FIG. 1

Vacuum-formed Plastazote shoes, with microcellular soles and heels. deformity it is a great comfort to obtain freedom from uppers over the foot, especially with the additional springiness of an inch of Plastazote underneath. In this patient there has been no sign of any ulceration at all since this support was supplied.

Patients with plantar ulceration are often in hospital for a considerable period simply because they cannot wear normal footwear. By using vacuum-formed Plastazote for the uppers and microcellular soles and heels directly attached to the Plastazote it is now possible to provide extraordinarily light and comfortable footwear, weighing for instance only $6 \mathrm{oz}(170 \mathrm{~g})$ for a pair of size 9 shoes (Fig. 1). Such footwear can be very quickly made, the vacuum forming being done over the cast or last of the affected foot. Most of the surgical footwear with leather uppers made in our workshops is made by the direct attachment method (not welted) and I have found that patients with partial amputations of the foot benefit considerably because the footwear made in this way is very much lighter than that made in the welted manner, and is also, by being firm but resilient, completely shock absorbing in walking.

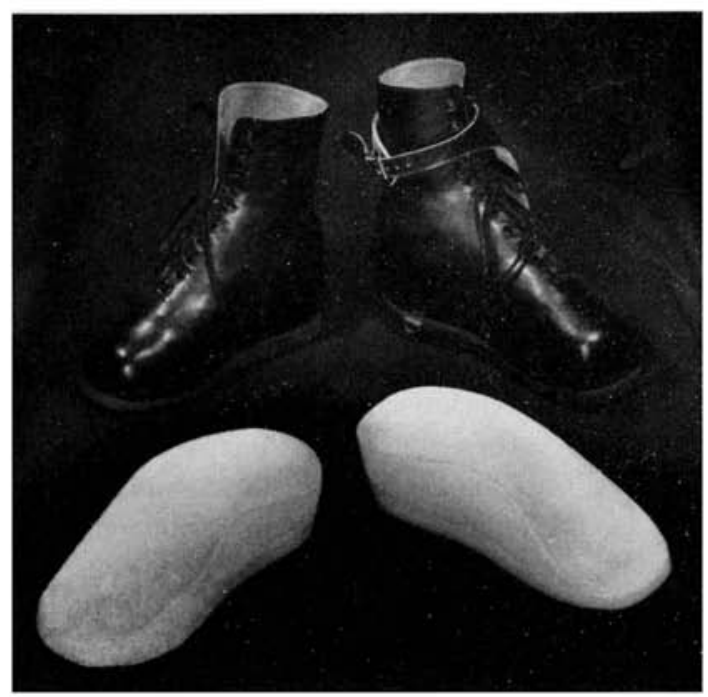

Fici. 2

Surgical boots and Plastazote insoles, for leprosy patient with grossly cleformed feet. 
To conclude, having described the old method of using sponge-rubber insoles with pads and toe blocks-until recently the best method available - which unfortunately did not entirely answer all the problems presented, there is no doubt that patients with deformed feet, prone to ulceration and with deformities that result in uneven balance, derive enormous benefit by having Plastazote insoles moulded directly to the base of their feet. This new method has been a major break-through in meeting the specialized requirements of these particular cases. Another advantage is that Plastazote is completely washable, although water re- pellent, and it can also be perforated for ventilation if required.

One important point worth stressing is that as Plastazote supports are less expense than normal supports and quick to make, they should always be supplied in duplicate to be worn on alternate days. It should also be emphasized that when Plastazote supports have been in use for a period of time, it is always necessary to reinforce the supports by adding another in $\frac{1}{4}$ in. $(0.5 \mathrm{~cm})$ thickness of Plastazote under the depressed area. This should be fixed to the original support by a rubber solution; the support itself need not be remoulded. 